PROCEEDINGS OF THE

AMERICAN MATHEMATICAL SOCIETY

Volume 137, Number 11, November 2009, Pages 3585-3592

S 0002-9939(09)09857-8

Article electronically published on July 1, 2009

\title{
ON THE IRREDUCIBLE REPRESENTATIONS OF A FINITE SEMIGROUP
}

\author{
OLEXANDR GANYUSHKIN, VOLODYMYR MAZORCHUK, AND BENJAMIN STEINBERG
}

(Communicated by Birge Huisgen-Zimmermann)

\begin{abstract}
Work of Clifford, Munn and Ponizovskiu parameterized the irreducible representations of a finite semigroup in terms of the irreducible representations of its maximal subgroups. Explicit constructions of the irreducible representations were later obtained independently by Rhodes and Zalcstein and by Lallement and Petrich. All of these approaches make use of Rees's theorem characterizing 0-simple semigroups up to isomorphism. Here we provide a short modern proof of the Clifford-Munn-Ponizovskii result based on a lemma of J. A. Green, which allows us to circumvent the theory of 0-simple semigroups. A novelty of this approach is that it works over any base ring.
\end{abstract}

\section{INTRODUCTION AND PRELIMINARIES}

Work of Clifford [5, 4, Munn [16, 15] and Ponizovskiu [17, parameterized the irreducible representations of a finite semigroup in terms of the irreducible representations of its maximal subgroups. (See [6, Chapter 5] for a full account of this work.) Explicit constructions of the irreducible representations were later obtained independently by Rhodes and Zalcstein 22 and by Lallement and Petrich 12 in terms of the Schützenberger representation by monomial matrices 23; see also 14 . All of these approaches make use of Rees's theorem [20 characterizing 0-simple semigroups up to isomorphism, thereby rendering the results somewhat inaccessible to the non-specialist in semigroup theory. As a consequence, it seems that when researchers from other areas need to use semigroup representation theory, they are forced to reinvent parts of the theory for themselves, e.g. 2, 3]. This paper, like [25, 26, 19, 13, 1], aims to reconcile semigroup representation theory with representation theory at large.

The goal of this note is to give a self-contained accounting of the theory of simple modules over the semigroup algebra of a finite semigroup using only the tools of associative algebras. This should make the results accessible to the general mathematician for the first time. Our key tool is a lemma of J. A. Green 10. An advantage of this approach is that it avoids Wedderburn theory and hence works over an arbitrary commutative ring with unit.

Received by the editors December 17, 2007, and, in revised form, November 28, 2008.

2000 Mathematics Subject Classification. Primary 16G10, 20M30, $20 \mathrm{M} 25$.

Key words and phrases. Representations, simple modules, semigroups.

The first author was supported in part by STINT.

The second author was supported in part by the Swedish Research Council.

The third author was supported in part by NSERC. 
We collect here some basic definitions and facts concerning finite semigroups that can be found in any of [11, 6, 21]. Let $S$ be a (fixed) finite semigroup. If $e$ is an idempotent, then $e S e$ is a monoid with identity $e$; its group of units $G_{e}$ is called the maximal subgroup of $S$ at $e$. Two idempotents $e, f$ are said to be isomorphic if there exist $x \in e S f$ and $x^{\prime} \in f S e$ such that $x x^{\prime}=e, x^{\prime} x=f$. In this case one can show that $e S e$ is isomorphic to $f S f$ as monoids and hence $G_{e} \cong G_{f}$.

If $s \in S$, then $J(s)=S^{1} s S^{1}$ is the principal (two-sided) ideal generated by $s$ (here $S^{1}$ means $S$ with an adjoined identity). Following Green [9], two elements $s, t$ of a semigroup $S$ are said to be $\mathscr{J}$-equivalent if $J(s)=J(t)$. In this case one writes $s \mathscr{J} t$. In fact there is a preorder on $S$ given by $s \leq \mathscr{J} t$ if $J(s) \subseteq J(t)$. This preorder induces an ordering on $\mathscr{J}$-classes in the usual way.

Fact 1. In a finite semigroup, idempotents $e, f$ are isomorphic if and only if $e \mathscr{J} f$, that is, $S e S=S f S$.

An element $s$ of a semigroup $S$ is said to be (von Neumann) regular if $s=s t s$ for some $t \in S$. Each idempotent is of course regular.

Fact 2. Let $S$ be a finite semigroup and $J$ a $\mathscr{J}$-class of $S$. Then the following are equivalent:

(1) $J$ contains an idempotent;

(2) $J$ contains a regular element;

(3) all elements of $J$ are regular;

(4) $J^{2} \cap J \neq \emptyset$.

A $\mathscr{J}$-class satisfying the equivalent conditions in Fact 2 is called a regular $\mathscr{J}$-class. The poset of regular $\mathscr{J}$-classes is denoted $\mathscr{U}(S)$. It was shown by Putcha [19] that over an algebraically closed field of characteristic zero, the module category of a regular semigroup (one in which all elements are regular) is a highest weight category [7] with weight poset $\mathscr{U}(S)$. We need one last fact about finite semigroups in order to state and prove the Clifford-Munn-Ponizovskiı theorem.

Fact 3. Let $S$ be a finite semigroup and $J$ a regular $\mathscr{J}$-class. Let $e \in J$ be an idempotent. Then $e S e \cap J=G_{e}$.

Let $J$ be a $\mathscr{J}$-class of $S$. Set $I_{J}=\{s \in S \mid J \nsubseteq J(s)\}$; it is the ideal of all elements of $S$ that are not $\mathscr{J}$-above some (equals any) element of $J$.

\section{Characterization and Construction of simple modules}

Fix a finite semigroup $S$ and a commutative ring with unit $K$. The semigroup algebra $K S$ need not be unital. If $A$ is a $K$-algebra, not necessarily unital, then by a simple module $M$, we mean a (right) $A$-module $M$ such that $M A \neq 0$ and $M$ contains no proper non-zero submodules or, equivalently, for all $0 \neq m \in M$, the cyclic module $m A=M$. Of course if $K$ is a field and $A$ is finite-dimensional, then every simple $A$-module is finite-dimensional, being cyclic and hence a quotient of the regular module $A$. The category of (right) $A$-modules will be denoted mod- $A$. We adopt the convention that if $A$ is unital, then by $\bmod -A$ we mean the category of unital $A$-modules. The reader should verify that all functors considered in this paper respect this convention.

If $M$ is a $K S$-module, then $\operatorname{Ann}_{S}(M)=\{s \in S \mid M s=0\}$. Clearly $\operatorname{Ann}_{S}(M)$ is an ideal of $S$. The following definition, due to Munn [16, is crucial to semigroup representation theory. 
Definition 4 (Apex). A regular $\mathscr{J}$-class $J$ is said to be the apex of a $K S$-module $M$ if $\operatorname{Ann}_{S}(M)=I_{J}$.

It is easy to see that $M$ has apex $J$ if and only if $J$ is the unique $\leq \mathscr{J}$-minimal $\mathscr{J}$-class that does not annihilate $M$.

Fix an idempotent transversal $E=\left\{e_{J} \mid J \in \mathscr{U}(S)\right\}$ to the set $\mathscr{U}(S)$ of regular $\mathscr{J}$-classes and set $G_{J}=G_{e_{J}}$. Let $A_{J}=K S / K I_{J}$. Notice that the category of $K S$-modules with apex $J$ can be identified with the full subcategory of $\bmod -A_{J}$ whose objects are modules $M$ such that $M e_{J} \neq 0$.

Our first goal is to show that every simple module has an apex. This result is due independently to Munn and Ponizovskiu [16, 15, 17.

Theorem 5. Let $M$ be a simple KS-module. Then $M$ has an apex.

Proof. Since $M K S \neq 0$, there is a $\leq \mathscr{J}$-minimal $\mathscr{J}$-class $J$ so that $J \nsubseteq \operatorname{Ann}_{S}(M)$. Let $I=S^{1} J S^{1}$; of course, $I$ is an ideal of $S$. Since $I \backslash J$ annihilates $M$ by minimality of $J$, it follows that $0 \neq M K J=M K I$. From the fact that $I$ is an ideal of $S$, we may deduce that $M K I$ is a $K S$-submodule and so by simplicity

$$
M=M K I=M K J .
$$

Therefore, since $J I_{J} \subseteq I \backslash J \subseteq \operatorname{Ann}_{S}(M)$, it follows from (2.1) that $I_{J}=\operatorname{Ann}_{S}(M)$. Now if $J$ is not regular, then Fact 2 implies $J^{2} \subseteq I \backslash J$ and hence $J$ annihilates $M$ by (2.1), a contradiction. Thus $J$ is regular and is an apex for $M$.

Now we wish to establish a bijection between simple $K S$-modules with apex $J$ and simple $K G_{J}$-modules. This relies on a well-known result of Green 10. Let $A$ be an algebra and $e$ an idempotent of $A$. Then $e A$ is an $e A e-A$-bimodule and $A e$ is an $A$-eAe-bimodule. Hence we have a restriction functor Res $: \bmod -A \rightarrow \bmod -e A e$ and induction/coinduction functors Ind, Coind $: \bmod -e A e \rightarrow \bmod -A$ given by

$$
\operatorname{Ind}(M)=M \otimes_{e A e} e A, \operatorname{Res}(M)=M e \text { and } \operatorname{Coind}(M)=\operatorname{Hom}_{e A e}(A e, M) .
$$

Moreover, Ind is right exact, Res is exact, Coind is left exact and Ind and Coind are the left and right adjoints of Res, respectively. This follows from observing that $\operatorname{Hom}_{A}(e A, M)=M e=M \otimes_{A} A e$ and the usual adjunction between hom and the tensor product. Moreover, it is well known that the unit of the first adjunction gives a natural isomorphism $M \cong \operatorname{Ind}(M) e$ while the counit of the second gives a natural isomorphism $\operatorname{Coind}(M) e \cong M$.

There are also two important functors $N, L: \bmod -A \rightarrow \bmod -A$ given by $N(M)=$ $\{m \in M \mid m A e=0\}$ and $L(M)=M e A$. It is easily verified that $N(M)$ is the largest $A$-submodule of $M$ that is annihilated by $e$, while $L(M)$ is the smallest $A$-submodule of $M$ with $L(M) e=M e$. Our next result can be found in [10, 6.2], but we reproduce the proof here for the convenience of the reader.

Lemma 6 (Green). Let $A$ be an algebra and e an idempotent of $A$.

(1) If $M$ is a simple $A$-module, then $M e=0$ or $M e$ is a simple eAe-module.

(2) If $V$ is a simple eAe-module, then $\operatorname{Ind}(V)$ has a unique maximal submodule $N(\operatorname{Ind}(V))$ and $\operatorname{Ind}(V) / N(\operatorname{Ind}(V))$ is the unique simple A-module $M$ with $M e \cong V$.

(3) If $V$ is a simple eAe-module, then $\operatorname{Coind}(V)$ has a unique minimal $A$ submodule $L(\operatorname{Coind}(V))$ and $L(\operatorname{Coind}(V))$ is the unique simple $A$-module $M$ with $M e \cong V$. 
Consequently, restriction yields a bijection between simple A-modules that are not annihilated by $e$ and simple eAe-modules.

Proof. To prove (1), assume $M e \neq 0$. Let $m \in M e$ be non-zero. Then $m e A=$ $m A=M$, so $m e A e=M e$. Thus $M e$ is simple.

Now we turn to (2). Let $V$ be a simple $e A e$-module and suppose $w \in \operatorname{Ind}(V)$ with $w \notin N(\operatorname{Ind}(V))$. Then $0 \neq w A e \subseteq \operatorname{Ind}(V) e$. But $\operatorname{Ind}(V) e \cong V$ is a simple $e A e$-module, so

$$
(w A e A e) A=\operatorname{Ind}(V) e A=\left(V \otimes_{e A e} e A\right) e A=\left(V \otimes_{e A e} e\right) A=\operatorname{Ind}(V)
$$

and thus $N(\operatorname{Ind}(V))$ is the unique maximal $A$-submodule of $\operatorname{Ind}(V)$. In particular, $\operatorname{Ind}(V) / N(\operatorname{Ind}(V))$ is a simple $A$-module. Since restriction is exact and $N(\operatorname{Ind}(V)) e=0$ by construction, it follows that

$$
[\operatorname{Ind}(V) / N(\operatorname{Ind}(V))] e \cong \operatorname{Ind}(V) e / N(\operatorname{Ind}(V)) e \cong \operatorname{Ind}(V) e \cong V .
$$

It remains to prove uniqueness. Suppose $M$ is a simple $A$-module such that $M e \cong V$. Then, using the adjunction between induction and restriction, we have $\operatorname{Hom}_{e A e}(V, M e) \cong \operatorname{Hom}_{A}\left(V \otimes_{e A e} e A, M\right)$. Hence the isomorphism $V \rightarrow M e$ corresponds to a non-zero homomorphism $\varphi: \operatorname{Ind}(V) \rightarrow M$, which is necessarily onto as $M$ is simple. But $N(\operatorname{Ind}(V))$ is the unique maximal submodule of $\operatorname{Ind}(V)$, so $\operatorname{ker} \varphi=N(\operatorname{Ind}(V))$ and hence $M \cong \operatorname{Ind}(V) / N(\operatorname{Ind}(V))$, as required.

Finally, to prove (3), first observe that if $M$ is any non-zero $A$-submodule of Coind $(V)$, then $M e \neq 0$. Indeed, suppose $M e=0$ and let $\varphi \in M$. Then, for any $x$ in $A e$, we have $\varphi(x)=(\varphi x e)(e)=0$ since $\varphi x e \in M e=0$. It follows that $M=0$. Since Coind $(V) e \cong V$ is a simple $e A e$-module, it now follows that if $M$ is a non-zero $A$-submodule of Coind $(V)$, then $M e=\operatorname{Coind}(V) e$ and hence

$$
L(\operatorname{Coind}(V))=\operatorname{Coind}(V) e A \subseteq M e A \subseteq M .
$$

This establishes that $L(\operatorname{Coind}(V))$ is the unique minimal $A$-submodule. Since $L(\operatorname{Coind}(V)) e=\operatorname{Coind}(V) e A e=\operatorname{Coind}(V) e \cong V$, it just remains to prove uniqueness. Suppose $M$ is a simple $A$-module with $M e \cong V$. Then the existence of a non-zero element of $\operatorname{Hom}_{e A e}(M e, V) \cong \operatorname{Hom}_{A}(M$, $\operatorname{Coind}(V))$ implies that $M$ admits a non-zero homomorphism to $\operatorname{Coind}(V)$. Hence $M$ is isomorphic to a simple $A$-submodule of $\operatorname{Coind}(V)$. But $L(\operatorname{Coind}(V))$ is the unique simple submodule of $\operatorname{Coind}(V)$ and so $M \cong L(\operatorname{Coind}(V))$, as required.

We may now complete the proof of the Clifford-Munn-Ponizovskiu theorem, with an explicit construction of the simple modules equivalent to the one found in [22, 12,

Theorem 7 (Clifford, Munn, Ponizovskiǔ). Let $S$ be a finite semigroup, $K$ a commutative ring with unit and $E=\left\{e_{J} \mid J \in \mathscr{U}(S)\right\}$ an idempotent transversal to the set $\mathscr{U}(S)$ of regular $\mathscr{J}$-classes of $S$. Let $G_{J}$ be the maximal subgroup $G_{e_{J}}$. Define functors $\operatorname{Ind}_{G_{J}}^{S}$, $\operatorname{Coind}_{G_{J}}^{S}: \bmod -K G_{J} \rightarrow \bmod -K S$ by

$$
\begin{aligned}
\operatorname{Ind}_{G_{J}}^{S}(V) & =V \otimes_{K G_{J}} e_{J}\left(K S / K I_{J}\right), \\
\operatorname{Coind}_{G_{J}}^{S}(V) & =\operatorname{Hom}_{K G_{J}}\left(\left(K S / K I_{J}\right) e_{J}, V\right) .
\end{aligned}
$$

Then:

(1) If $M$ is a simple $K S$-module with apex $J$, then $M e_{J}$ is a simple $K G_{J^{-}}$ module. 
(2) If $V$ is a simple $K G_{J}$-module, then

$$
N=\left\{w \in \operatorname{Ind}_{G_{J}}^{S}(V) \mid w K S e_{J}=0\right\}
$$

is the unique maximal $K S$-submodule of $\operatorname{Ind}_{G_{J}}^{S}(V)$ and $\operatorname{Ind}_{G_{J}}^{S}(V) / N$ is the unique simple $K S$-module $M$ with apex $J$ such that $M e_{J}=V$.

(3) If $V$ is a simple $K G_{J}$-module, then $\operatorname{Coind}_{G_{J}}^{S}(V) e A$ is the unique minimal $A$-submodule of $\operatorname{Coind}_{G_{J}}^{S}(V)$ and moreover is the unique simple $K S$-module $M$ with apex $J$ such that $M e_{J}=V$.

Consequently, if $K$ is a field, there is a bijection between irreducible representations of $S$ and irreducible representations of the maximal subgroups $G_{J}$ of $S, J \in \mathscr{U}(S)$.

Proof. Theorem 5 implies that every simple $K S$-module $M$ has an apex. Again setting $A_{J}=K S / I_{J}$ for a regular $\mathscr{J}$-class $J$, we know that simple $K S$-modules with apex $J$ are in bijection with simple $A_{J}$-modules $M$ such that $M e_{J} \neq 0$. It follows directly from Fact 3 that

$$
e_{J} A_{J} e_{J}=K e_{J} S e_{J} / K e_{J} I_{J} e_{J}=K G_{J} .
$$

Lemma 6 then yields that simple $A_{J}$-modules not annihilated by $e_{J}$, that is, simple $K S$-modules with apex $J$, are in bijection with simple $K G_{J}$-modules in the prescribed manner.

Let us make a remark to relate the above construction of the simple modules to the ones found in 22,12 . All the facts about finite semigroups used in this discussion can be found in the appendix of 21] or in 11. According to Green 9], two elements $s, t$ of a semigroup are said to be $\mathscr{R}$-equivalent if they generate the same principal right ideal. Dually $s, t$ are said to be $\mathscr{L}$-equivalent if they generate the same principal left ideal. If $S$ is a finite semigroup, then it is well known (retaining our previous notation) that $e_{J} S \cap J$ is the $\mathscr{R}$-class $R_{e_{J}}$ of $e_{J}$ and $S e_{J} \cap J$ is the $\mathscr{L}$-class $L_{e_{J}}$ of $e_{J}$. Furthermore, left multiplication yields a free action of $G_{J}$ on the left of $R_{e_{J}}$ by automorphisms of the action of $S$ on the right of $R_{e_{J}}$ by partial transformations (induced by right multiplication). Moreover, the $G_{J}$-orbits on $R_{e_{J}}$ are in bijection with the set of $\mathscr{L}$-classes of $J$. Let $T$ be a transversal to the $G_{J}$-orbits. Now $e_{J} K S / I_{J}$ can be identified as a vector space with $K R_{e_{J}}$ and the right $K S$-module structure is the linearization of the right action of $S$ on $R_{e_{J}}$ described above. Moreover, under this identification, the left $K G_{J}$-module structure on $K R_{e_{J}}$ is induced by the free left action of $G_{J}$ on $R_{e_{J}}$ and so $K R_{e_{J}}$ is a free left $K G_{J}$-module with basis $T$. In particular, the functor $\operatorname{Ind}_{G_{J}}^{S}$ is exact. It is straightforward to show using $[8$, Theorem 10.4.1] that under the usual identification of maximal subgroups inside $J$, the $K G_{J}-K S$-bimodule $K R_{e_{J}}$ does not depend (up to isomorphism) on the choice of $e_{J} \in J$.

From the above it follows that $\operatorname{End}_{K G_{J}}\left(K R_{e_{J}}\right) \cong M_{n}\left(K G_{J}\right)$, where $n$ is the number of $\mathscr{L}$-classes in $J$, and so there results a representation $\rho_{J}: S \rightarrow M_{n}\left(K G_{J}\right)$, which is easily checked to be the classical right Schützenberger representation by row monomial matrices [6, 23] since if $s \in S$ and $t \in T$, then either $t s=0$ or $t s=g t^{\prime}$ for unique elements $t^{\prime} \in T$ and $g \in G_{J}$.

Now let $V$ be a simple $K G_{J}$-module affording the irreducible representation $\varphi: G_{J} \rightarrow G L_{r}(K)$. Then the matrix representation afforded by the module $V \otimes_{K G_{J}} K R_{e_{J}}$ is the tensor product of $\varphi$ with $\rho_{J}$. Now an element of $S e_{J}$ which 
does not belong to $J$ automatically annihilates $V \otimes_{K G_{J}} K R_{e_{J}}$, so the unique maximal submodule consists of those vectors annihilated by $S e_{J} \cap J=L_{e_{J}}$, the $\mathscr{L}$-class of $e_{J}$. If one chooses Rees matrix coordinates for $J$ [6, 21], then it is not hard to show that the vectors annihilated by the $\mathscr{L}$-class of $e_{J}$ are those belonging to the null space of the image of the sandwich matrix under $\varphi$. Hence the construction of the simple modules we have provided corresponds exactly to the construction found in 22, 12, but our proof avoids Rees matrix semigroups and Munn algebras.

The coinduced module also has a natural semigroup theoretic interpretation. Indeed, there is an isomorphism of $K S$-modules,

$$
\operatorname{Hom}_{K G_{J}}\left(\left(K S / K I_{J}\right) e_{J}, V\right) \cong \operatorname{Hom}_{G_{J}}\left(L_{e_{J}}, V\right),
$$

where we view $L_{e_{J}}$ and $V$ as right $G_{J}$-sets and the right-hand side of (2.2) is made a vector space via pointwise operations. The semigroup $S$ acts on the left of $L_{e_{J}}$ by the left Schützenberger representation, and this induces the $K S$-module structure on $\operatorname{Hom}_{G_{J}}\left(L_{e_{J}}, V\right)$. Since $L_{e_{J}}$ is a free right $G_{e_{J}}$-set and the orbits are in bijection with the set of $\mathscr{R}$-classes in $J$, elements of $\operatorname{Hom}_{G_{J}}\left(L_{e_{J}}, V\right)$ are in bijection with elements of $V^{m}$, where $m$ is the number of $\mathscr{R}$-classes of $J$. The space $V^{m}$ (viewed as row vectors) is naturally a right $K S$-module via the left Schützenberger representation $\lambda_{J}: S \rightarrow M_{m}\left(K G_{J}\right)$ and the module structure agrees with the original one. If one chooses Rees matrix coordinates for $J$ [6, 21, then right multiplication by the structure matrix $C$ takes $V^{n}$ to $V^{m}$, where $n$ is the number of $\mathscr{L}$-classes of $S$. One can verify that the image of $C$ is the unique minimal $K S$-submodule of $V^{m}$. (The fact that it is a submodule is a consequence of the so-called linked equations [11, 21].) This yields the other construction of the irreducible representations found in [22]. Since $L_{e_{J}}$ is a free right $G_{J}$-set, it follows that $\operatorname{Coind}_{G_{J}}^{S}$ is exact. It should be mentioned that all coinduced constructions can be obtained from induced constructions for the opposite semigroup via duality. A more detailed analysis from this viewpoint can be found in 13.

Putcha has used both the induced and coinduced modules, which he calls the left and right induced modules, in his work on representation theory [19, 18].

As an application, we provide the description of the irreducible representations of an idempotent semigroup that was rediscovered by Brown 2, 3. and put to good effect in the study of random walks. First we establish a well-known lemma.

Lemma 8. Let $S$ be a semigroup of idempotents and let $J$ be a $\mathscr{J}$-class of $S$. Then the complement of $I_{J}$ is a subsemigroup of $S$.

Proof. First we show that $J$ is a subsemigroup. Let $e, f \in J$. Then we have $e=u f v$ for some $u, v \in S$ and so efv $=u f v f v=u f v=e$, establishing $e f \in J$. Next suppose $J \subseteq S s S \cap S s^{\prime} S$. We need $J \subseteq S s s^{\prime} S$. Let $e \in J$. Then $e=u s v$ and $e=u^{\prime} s^{\prime} v^{\prime}$ with $u, v, u^{\prime}, v^{\prime} \in S$. Since $u s(v u s) v=e$ and $u^{\prime}\left(s^{\prime} v^{\prime} u^{\prime}\right) s^{\prime} v^{\prime}=e$, it follows that vus $\mathscr{J}$ e $\mathscr{J} s^{\prime} v^{\prime} u^{\prime}$. Since $J$ is a subsemigroup, $v u s s^{\prime} v^{\prime} u^{\prime} \in J$ and hence $J \subseteq S s s^{\prime} S$, as required.

Corollary 9. Let $S$ be a finite semigroup all of whose elements are idempotents. Then all the irreducible representations of $S$ over a field have degree one and the unique irreducible representation $\varphi_{J}$ with apex $J$ is given by

$$
\varphi_{J}(s)= \begin{cases}0 & s \in I_{J}, \\ 1 & \text { otherwise }\end{cases}
$$


Proof. Let $J$ be a regular $\mathscr{J}$-class. Lemma 8 implies that (2.3) is an irreducible representation with apex $J$. It is afforded by $K$ with $S$-action

$$
k s= \begin{cases}0 & s \in I_{J} \\ k & \text { otherwise. }\end{cases}
$$

Since $G_{J}$ is trivial, there is exactly one simple $K S$-module with apex $J$, namely the quotient of $M=e_{J} K S / K I_{J}$ by its unique maximal submodule $N$. Now $R_{e_{J}}=$ $e_{J} S \backslash I_{J}=e_{J} S \cap J$ is a basis for $M$. As a consequence of Lemma $8, R_{e_{J}} s \subseteq R_{e_{J}}$ for $s \in S \backslash I_{J}$ and $R_{e_{J}} s \subseteq I_{J}$ otherwise. Thus the augmentation map $\varepsilon: M \rightarrow K$ sending each element of $R_{e_{J}}$ to 1 is a surjective morphism of $K S$-modules with kernel the unique maximal submodule $N$ of $M$, as $K$ is of course simple. This completes the proof.

The above argument applies mutatis mutandis to semigroups all of whose subgroups are trivial and whose regular $\mathscr{J}$-classes are subsemigroups. This class of semigroups, known as DA, was introduced by Schützenberger in his study of unambiguous products of regular languages [24].

\section{REFERENCES}

[1] J. Almeida, S. Margolis, B. Steinberg, and M. Volkov. Representation theory of finite semigroups, semigroup radicals and formal language theory. Trans. Amer. Math. Soc., 361(3):1429-1461, 2009. MR2457405

[2] K. S. Brown. Semigroups, rings, and Markov chains. J. Theoret. Probab., 13(3):871-938, 2000. MR.1785534 (2001e:60141)

[3] K. S. Brown. Semigroup and ring theoretical methods in probability. In Representations of finite dimensional algebras and related topics in Lie theory and geometry, volume 40 of Fields Inst. Commun., pages 3-26. Amer. Math. Soc., Providence, RI, 2004. MR2057147 (2005b:60118)

[4] A. H. Clifford. Matrix representations of completely simple semigroups. Amer. J. Math., 64:327-342, 1942. MR0006551(4:4a)

[5] A. H. Clifford. Basic representations of completely simple semigroups. Amer. J. Math., 82:430-434, 1960. MR0116062 (22:6857)

[6] A. H. Clifford and G. B. Preston. The algebraic theory of semigroups. Vol. I. Mathematical Surveys, No. 7. American Mathematical Society, Providence, RI, 1961. MR.0132791 (24:A2627)

[7] E. Cline, B. Parshall, and L. Scott. Finite-dimensional algebras and highest weight categories. J. Reine Angew. Math., 391:85-99, 1988. MR961165 (90d:18005)

[8] O. Ganyushkin and V. Mazorchuk. Classical finite transformation semigroups, an introduction. Number 9 in Algebra and Applications. Springer, 2009. MR.2460611

[9] J. A. Green. On the structure of semigroups. Ann. of Math. (2), 54:163-172, 1951. MR0042380(13:100d)

[10] J. A. Green. Polynomial representations of $\mathrm{GL}_{n}$, volume 830 of Lecture Notes in Mathematics. Springer-Verlag, Berlin, 1980. MR606556 (83j:20003)

[11] K. Krohn, J. Rhodes, and B. Tilson. Algebraic theory of machines, languages, and semigroups. Edited by Michael A. Arbib. With a major contribution by Kenneth Krohn and John L. Rhodes. Academic Press, New York, 1968, Chapters 1, 5-9. MR0232875 (38:1198)

[12] G. Lallement and M. Petrich. Irreducible matrix representations of finite semigroups. Trans. Amer. Math. Soc., 139:393-412, 1969. MR0242973 (39:4300)

[13] S. W. Margolis and B. Steinberg. The quiver of an algebra associated to the MantaciReutenauer descent algebra and the homology of regular semigroups. Algebr. Represent. Theory, to appear.

[14] D. B. McAlister. Characters of finite semigroups. J. Algebra, 22:183-200, 1972. MR0301125 $(46: 283)$

[15] W. D. Munn. On semigroup algebras. Proc. Cambridge Philos. Soc., 51:1-15, 1955. MR0066355 (16:561c) 
[16] W. D. Munn. Matrix representations of semigroups. Proc. Cambridge Philos. Soc., 53:5-12, 1957. MR0082050(18:489g)

[17] I. S. Ponizovskil. On matrix representations of associative systems. Mat. Sb. N.S., 38(80):241260, 1956. MR0081292(18:378d)

[18] M. S. Putcha. Complex representations of finite monoids. Proc. London Math. Soc. (3), 73(3):623-641, 1996. MR1407463 (97e:20093)

[19] M. S. Putcha. Complex representations of finite monoids. II. Highest weight categories and quivers. J. Algebra, 205(1):53-76, 1998. MR1631310 (99f:20106)

[20] D. Rees. On semi-groups. Proc. Cambridge Philos. Soc., 36:387-400, 1940. MR0002893 $(2: 127 \mathrm{~g})$

[21] J. Rhodes and B. Steinberg. The q-theory of finite semigroups. Springer Monographs in Mathematics. Springer, New York, 2009. MR2472427

[22] J. Rhodes and Y. Zalcstein. Elementary representation and character theory of finite semigroups and its application. In Monoids and semigroups with applications (Berkeley, CA, 1989), pages 334-367. World Sci. Publ., River Edge, NJ, 1991. MR.1142387 (92k:20129)

[23] M.-P. Schützenberger. Sur la représentation monomiale des demi-groupes. C. R. Acad. Sci. Paris, 246:865-867, 1958. MR0095886 (20:2384)

[24] M. P. Schützenberger. Sur le produit de concaténation non ambigu. Semigroup Forum, 13(1):47-75, 1976/77. MR0444824 (56:3171)

[25] B. Steinberg. Möbius functions and semigroup representation theory. J. Combin. Theory Ser. A, 113(5):866-881, 2006. MR2231092(2007c:20144)

[26] B. Steinberg. Möbius functions and semigroup representation theory. II. Character formulas and multiplicities. Adv. Math., 217(4):1521-1557, 2008. MR2382734 (2009e:20131)

Department of Mechanics and Mathematics, Kyiv Taras Shevchenko University, 64, Volodymyrska Street, 01033, Kyiv, Ukraine

E-mail address: ganiyshk@univ.kiev.ua

Department of Mathematics, Uppsala University, SE 471 06, Uppsala, Sweden - and - Department of Mathematics, University of Glasgow, University Gardens, GlasGOW G12 8QW, United Kingdom

E-mail address: mazor@math.uu.se

E-mail address: v.mazorchuk@maths.gla.ac.uk

School of Mathematics and Statistics, Carleton University, 1125 Colonel By Drive, Ottawa, Ontario K1S 5B6, Canada

E-mail address: bsteinbg@math.carleton.ca 